\title{
gु \\ Anomalous Hall effect in Fe (001) epitaxial thin films over a wide range in conductivity
}

\author{
S. Sangiao, ${ }^{1}$ L. Morellon, ${ }^{1,2, *}$ G. Simon, ${ }^{1}$ J. M. De Teresa, ${ }^{2}$ J. A. Pardo, ${ }^{3}$ J. Arbiol,,${ }^{4}$ and M. R. Ibarra ${ }^{1,2}$ \\ ${ }^{1}$ Departamento de Física de la Materia Condensada and Instituto de Nanociencia de Aragón, Universidad de Zaragoza, \\ Interfacultades II, Zaragoza 50009, Spain \\ ${ }^{2}$ Departamento de Física de la Materia Condensada and Instituto de Ciencia de Materiales de Aragón, Facultad de Ciencias, \\ Universidad de Zaragoza-CSIC, Zaragoza 50009, Spain \\ ${ }^{3}$ Departamento de Ciencia y Tecnología de Materiales y Fluidos and Instituto de Nanociencia de Aragón, Universidad de Zaragoza, \\ Zaragoza 50018, Spain \\ ${ }^{4}$ TEM-MAT, Serveis Cientificotècnis, Universitat de Barcelona, 08028 Barcelona, Spain
}

(Received 28 October 2008; published 23 January 2009)

\begin{abstract}
We report Hall-effect measurements of epitaxial Fe (001) thin films grown on $\mathrm{MgO}(001)$. We have focused on the dependence of the anomalous Hall effect (AHE) in heteroepitaxial structures $\mathrm{MgO}(001) / / \mathrm{Fe}(t) / \mathrm{MgO}$ with $t=10,2.5,2,1.8$, and $1.3 \mathrm{~nm}$. Our results have been interpreted in terms of a recent unified theory of the AHE. We have demonstrated that the thickness and roughness of the Fe layer are control parameters to tune both the longitudinal conductivity $\sigma_{x x}$ and anomalous Hall conductivity $\sigma_{x y}$. In this way, we report a crossover from the intrinsic moderately dirty region of conductivities where $\sigma_{x y}=$ const to the dirty region of poorly conducting materials $\left(\sigma_{x x}<10^{4} \mathrm{~S} / \mathrm{cm}\right)$ where we have found that the relation $\sigma_{x y} \propto \sigma_{x x}^{n}$ with $n=1.66(4)$ holds, in good agreement, with the expected universal scaling relationship reported in other ferromagnetic compounds.
\end{abstract}

DOI: 10.1103/PhysRevB.79.014431

PACS number(s): 75.47.- m, 73.50.Jt, 75.50.Bb

\section{INTRODUCTION}

The Hall resistivity in ferromagnetic materials, $\rho_{x y}$, is given by the empirical relation $\rho_{x y}=R_{O} \mu_{0} H+R_{S} \mu_{0} M$, where $H$ is the applied magnetic field and $M$ is the spontaneous magnetization. The first term describes the ordinary Hall effect (OHE) and the second, in general much larger than the first one, is the anomalous Hall effect (AHE). The OHE is caused by the Lorentz force acting on moving charged carriers, whereas the origin of the AHE has been a controversial and intriguing issue for decades, this effect being explained by theories based on intrinsic ${ }^{1-3}$ or extrinsic contributions. ${ }^{4-7}$ Depending on its origin, different dependences between $\rho_{x y}$ (anomalous part) and the longitudinal resistivity, $\rho_{x x}$, are given: for a dominating intrinsic mechanism based on the Berry phase of Bloch waves, a dependence $\rho_{x y} \propto \rho_{x x}^{2}$ is followed, whereas for the skew scattering or the side jump, $\rho_{x y} \propto \rho_{x x}$ or $\rho_{x y} \propto \rho_{x x}^{2}$ are expected, respectively. In a recent publication, ${ }^{8}$ a unified theory based on multiband ferromagnetic metals with dilute impurities taking on an equal footing both intrinsic and extrinsic contributions has been proposed. Three regimes can be distinguished as a function of the longitudinal conductivity, $\sigma_{x x}$. In the clean limit of extremely high conductivity, the skew scattering causes the effect $\left(\sigma_{x y} \propto \sigma_{x x}\right)$. An extrinsic-to-intrinsic crossover occurs at lower conductivities $\left(\sigma_{x x}=10^{4}-10^{6} \mathrm{~S} / \mathrm{cm}\right)$, where $\sigma_{x y}$ $=$ const. In the dirty regime $\left(\sigma_{x x}<10^{4} \mathrm{~S} / \mathrm{cm}\right)$ a scaling relation $\sigma_{x y} \propto \sigma_{x x}^{1.6}$ is predicted, which is caused by the damping of the intrinsic contribution. This scaling has been experimentally found in a large series of low-conductivity compounds irrespective of hopping or metallic conduction, ${ }^{9}$ intriguingly suggesting a universal scaling behavior. ${ }^{10-13}$

To our knowledge no single material has been found to span all three regimes proposed in Ref. 8. In this paper we will demonstrate that this is possible in pure bcc Fe (001) epitaxial thin films by adequately engineering the conductivity. Within this scope, we expect that Hall-effect measurements of $\mathrm{Fe}(001)$ ultrathin films can extend previous existing results into the dirty regime, therefore providing a comprehensive view and interpretation of the AHE in bcc Fe, and giving additional support to the unified picture proposed in Ref. 8. Besides, transport properties of ultrathin films and surfaces play an important role for the development of nanoscaled electronic devices. We have chosen $\mathrm{MgO} / / \mathrm{Fe} /$ $\mathrm{MgO}$ as an ideal system because of both the theoretical predictions on the extremely low $\mathrm{Fe}-\mathrm{MgO}$ interaction at the interface and the giant magnetic moment predicted for a monolayer of $\mathrm{Fe}$ adsorbed on $\mathrm{MgO}(001),{ }^{14}$ as well as the interest in applications for $\mathrm{Fe} / \mathrm{MgO} / \mathrm{Fe}$-based magnetic tunnel junctions. ${ }^{15}$ Of particular interest is the effect of the reduction in the dimensionality in the nature of the conduction mechanisms and its impact on the magnetotransport. ${ }^{16}$ The first experiments in Fe ultrathin films grown on $\mathrm{MgO}(001)$ were reported by Liu et al.,${ }^{17}$ finding a minimum in the temperature dependence of the resistivity that was attributed to a crossover from two-dimensional (2D) disordered to threedimensional (3D) metallic behavior. An interesting enhanced and oscillatory magnetoresistance of thin $\mathrm{Fe}(001)$ films has also been recently reported. ${ }^{18}$

\section{EXPERIMENT}

We have grown epitaxial $\mathrm{Fe}$ thin films on single-crystal $\mathrm{MgO}$ (001) substrates in a combined pulsed laser deposition (PLD)-sputtering system (Neocera Llc) with a base pressure $<5 \times 10^{-9}$ Torr. The $\mathrm{Fe}$ layer was dc sputtered from a pure $(99.99 \%)$ target and protected from ambient oxidation by means of $\approx 3 \mathrm{~nm} \mathrm{MgO}$ overlayer, grown by $\mathrm{rf}$ sputtering from a stoichiometric polycrystalline target. The 
substrate temperature was kept at $200{ }^{\circ} \mathrm{C}$ during deposition. The thickness of the Fe layer was varied in the range $t=3$ $-1 \mathrm{~nm}$ and optimization of the growth conditions has been carried out on a $t=10 \mathrm{~nm}$ sample. The $\mathrm{MgO}(001) / / \mathrm{Fe}(t) / \mathrm{MgO}(3 \mathrm{~nm})$ heterostructures have been characterized by x-ray diffraction (XRD) $(\theta-2 \theta, \omega$ scans, $\phi$ scans) and X-ray reflectivity (XRR), high-resolution transmission electron microscopy (HRTEM), vibrating-sample magnetometer (VSM), and superconducting quantum interference device (SQUID) magnetometry. We have used a dual-beam equipment that integrates a $30 \mathrm{kV}$ field-emission electron column and a Ga-based $30 \mathrm{kV}$ ion column to prepare electron-transparent lamellae $(<100 \mathrm{~nm})$ out of thin films for subsequent cross-section transmission electron microscopy studies. An omniprobe nanomanipulator was used to place the lamellae on suitable $\mathrm{Cu}$ transmission electron microscopy (TEM) grids. HRTEM images were obtained in a field-emission-gun microscope JEOL 2010F working at 200 $\mathrm{kV}$ and with a point resolution of $0.19 \mathrm{~nm}$. Electrical transport and Hall-effect measurements as a function of temperature were performed in square $\left(5 \times 5 \mathrm{~mm}^{2}\right)$ samples in the Van-der-Pauw geometry in a commercial physical properties measurement system (PPMS) in the temperature range from 300 down to $2 \mathrm{~K}$ and in magnetic fields up to $90 \mathrm{kOe}$. In Hall-effect measurements, the contribution from offset values or magnetoresistance was carefully removed by subtracting $\rho_{x y}(-H)$ from $\rho_{x y}(H)$. We have also performed some electrical transport measurements on patterned samples of $t$ $\cong 10 \mathrm{~nm}$ by means of a two-step optical lithography process. The typical electrodes obtained for the flow of the current were either 300 or $4 \mu \mathrm{m}$ wide and pads were patterned for the measurements of the voltage drop. This provides a welldefined geometry minimizing offset voltages in the measurements.

\section{RESULTS AND DISCUSSION}

In order to optimize the growth conditions, we have started with thicker Fe samples $(t=10 \mathrm{~nm})$. High resolution XRD measurements and HRTEM (not shown) reveal an epitaxial growth with the expected out-of-plane (001) orientation with a $45^{\circ}$ rotation of the in-plane axes: $\mathrm{MgO}(001)$ $[100] / / \mathrm{Fe}(001)[110]$. The mosaic spread is typically $\Delta \omega$ $(002)=1.2^{\circ}$ and XRR fits yield an rms roughness of $\sim 0.2 \mathrm{~nm}$. Magnetization measurements show the expected in-plane magnetic anisotropy with the easy axis along the $\mathrm{Fe}$ [100] direction and a coercivity field $<10$ Oe. All these results are in good agreement with published data in thin $\mathrm{Fe}$ films of similar thickness grown by means of molecular beam epitaxy (MBE), ${ }^{19}$ ultra high vacuum (UHV) sputtering, ${ }^{20}$ and PLD. ${ }^{21}$

We show in Fig. 1 the temperature dependence of the electrical resistivity for two selected samples with $t=2.5$ and $1.8 \mathrm{~nm}$. For the ultrathin films $t<3 \mathrm{~nm}$, the values of the thickness and interface roughness have been determined by fitting the experimental XRR profiles by means of the LEPTOS software (BRUKER AXS). The room-temperature resistivity values are enhanced as compared to those of thicker samples $(13.5 \mu \Omega \mathrm{cm}$ for $t=10 \mathrm{~nm} ; 9.7 \mu \Omega \mathrm{cm}$ for bulk iron) as expected from the increase in surface scattering (de-

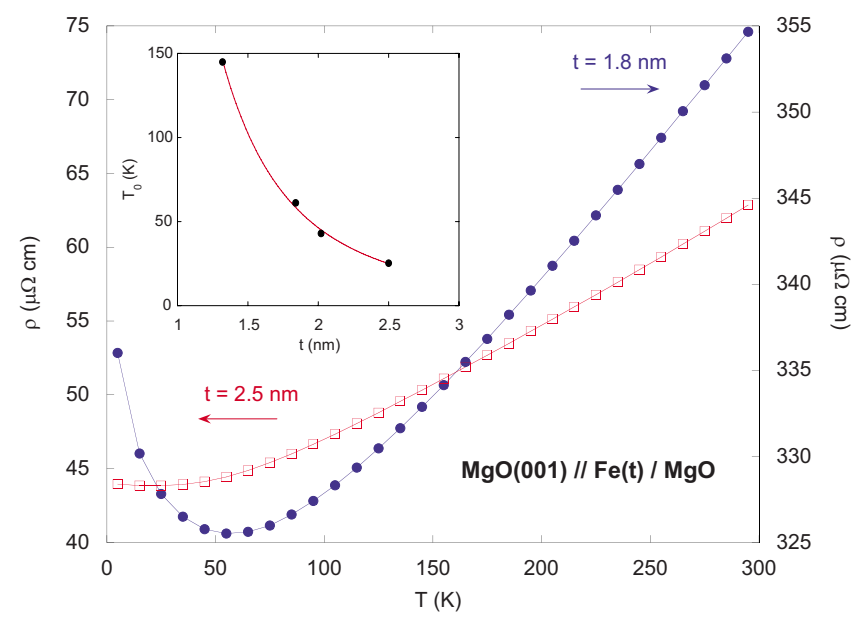

FIG. 1. (Color online) Temperature dependence of the resistivity, $\rho$, of $\mathrm{MgO}(001) / / \mathrm{Fe}(t) / \mathrm{MgO}$ with $t=2.5 \mathrm{~nm}$ (open squares) and $t=1.8 \mathrm{~nm}$ (block circles). The inset shows the thickness dependence of $T_{0}$, defined as the temperature of the minimum in $\rho(T)$.

crease in thickness) as qualitatively described by the FuchsSondheimer equation. ${ }^{22}$ It is interesting to note that the room-temperature resistivity values in our ultrathin samples as a function of thickness exceed those expected from the Fuchs-Sondheimer equation as also observed in Ref. 23. An interesting feature is a shallow minimum that starts to develop at very low temperature in the $2.5 \mathrm{~nm}$ sample, and is clearly seen in that of $1.8 \mathrm{~nm}$. The temperature of the minimum $T_{0}$ as a function of thickness is displayed in the inset of Fig. 1. These results are in good agreement with those observed by other authors, ${ }^{17,18,23}$ which have been related to a $2 \mathrm{D}$ to $3 \mathrm{D}$ transition and explained in terms of electron localization and/or electron-electron interaction effects. ${ }^{16,23}$ Therefore, we propose that the thickness of the Fe layer can be utilized as a control parameter of the conductivity of bcc Fe. In the following, we will focus on the Hall effect.

We have measured the Hall resistivity, $\rho_{x y}$, as a function of temperature in the 300-2 $\mathrm{K}$ range and in magnetic fields of up to $90 \mathrm{kOe}$ in several ultrathin samples of thickness $t$ $=2.5,2.0,1.8$, and $1.3 \mathrm{~nm}$, and in a thicker patterned sample of $t=10 \mathrm{~nm}$. When working with such thin samples the quality of the samples in terms of continuity of the different layers and crystallinity has to be addressed. In Fig. 2, the

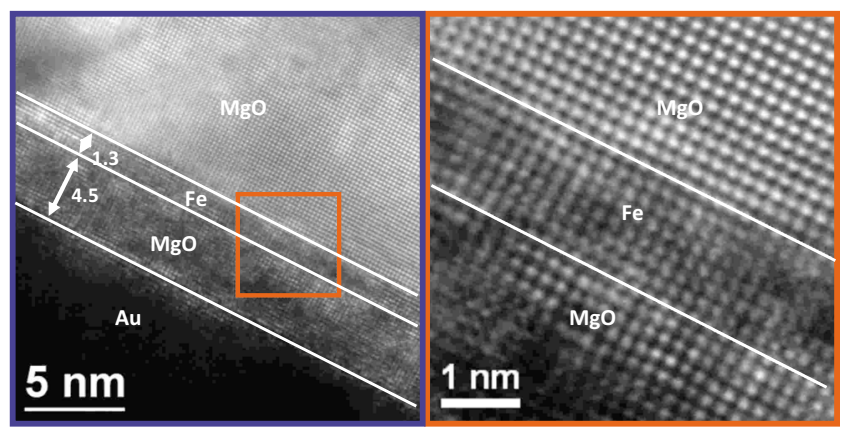

FIG. 2. (Color online) Cross sectional HRTEM micrograph of a $\mathrm{MgO}(001) / / \mathrm{Fe}(t=1.3 \mathrm{~nm}) / \mathrm{MgO}$ thin film (left). The interface region marked has been zoomed in (right). 

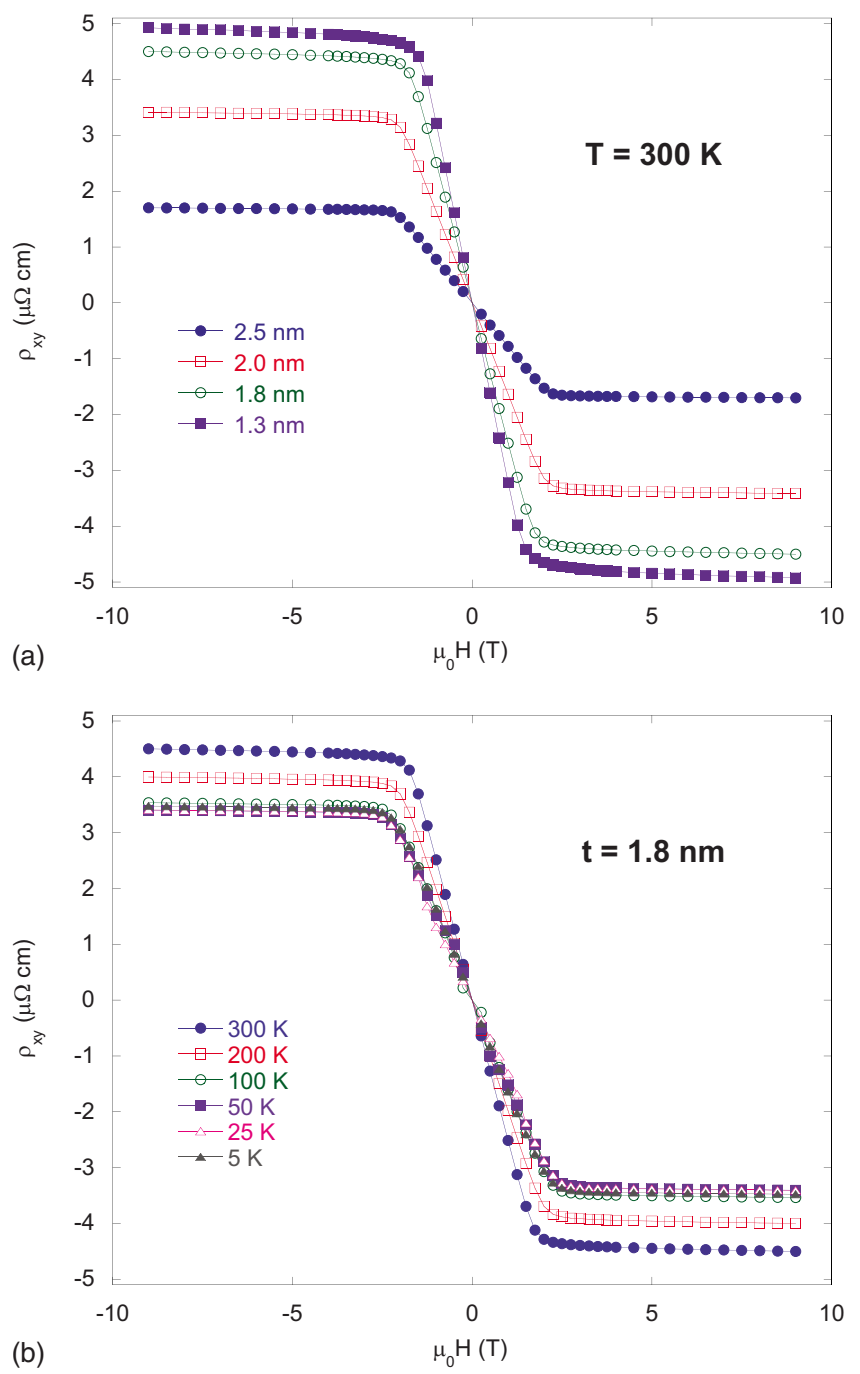

FIG. 3. (Color online) (a) Thickness dependence of the roomtemperature Hall resistivity, $\rho_{x y}$, of $\mathrm{MgO}(001) / / \mathrm{Fe}(t) / \mathrm{MgO}$ with $t=2.5,2.0,1.8$, and $1.3 \mathrm{~nm}$. In (b), $\rho_{x y}$ at selected temperatures is shown for a $\mathrm{MgO}(001) / / \mathrm{Fe}(t=1.8 \mathrm{~nm}) / \mathrm{MgO}$ sample.

HRTEM cross-section image of the thinnest sample with $t$ $=1.3 \mathrm{~nm}$ can be seen (left) together with a blow up of the interface region (right). From the analysis of the HRTEM data we can demonstrate that $\mathrm{Fe}$ grows epitaxially on $\mathrm{MgO}$ according to the expected out-of-plane (001) orientation with a $45^{\circ}$ rotation of the in-plane axes: $\mathrm{MgO}(001)[100] / / \mathrm{Fe}$ (001) [110]. The MgO capping layer also grows epitaxially on Fe following the same epitaxial relationship as that of the Fe layer onto the $\mathrm{MgO}$ substrate. We have also performed a study of the dislocation density at the $\mathrm{MgO}$ substrate- $-\mathrm{Fe}$ layer interface, with this being very low probably due to the fully strained state of the Fe layer ${ }^{24}$ in contrast to the case of much thicker samples. In Fig. 3, we show the field dependence of $\rho_{x y}$ for different samples at $T=300 \mathrm{~K}$ [Fig. 3(a)] and for a selected sample of $t=1.8 \mathrm{~nm}$ at several temperatures [Fig. 3(b)]. As can be seen in Fig. 3(a) the roomtemperature anomalous Hall resistivity strongly depends on the thickness of the Fe layer increasing from $1.66 \mu \Omega \mathrm{cm}$ for $\mathrm{t}=2.5 \mathrm{~nm}$ up to $4.74 \mu \Omega \mathrm{cm}$ for $\mathrm{t}=1.3 \mathrm{~nm}$, i.e., almost a factor of three. As we will see later, this results in a factor

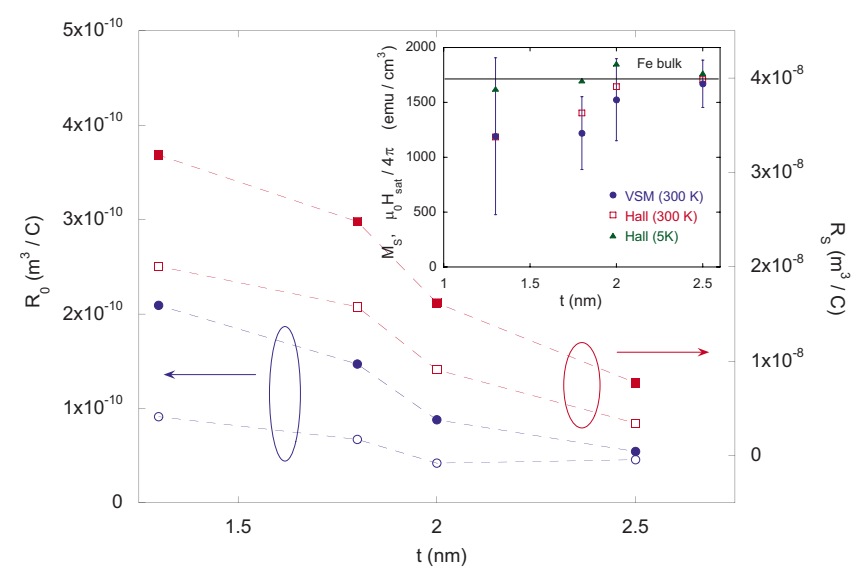

FIG. 4. (Color online) Thickness dependence of the OHE coefficient, $R_{O}$, (circles) and AHE coefficient, $R_{S}$, (squares) of $\mathrm{MgO}(001) / / \mathrm{Fe}(t) / \mathrm{MgO}$ with $t=2.5,2.0,1.8$, and $1.3 \mathrm{~nm}$ at room temperature (open symbols) and $T=5 \mathrm{~K}$ (block symbols). The inset displays the thickness dependence of the room-temperature saturation magnetization, $M_{S}$, from VSM magnetometry (block circles) together with that determined from the saturation field of the Hall resistivity isotherms, $\mu_{0} H_{\mathrm{sat}} / 4 \pi$.

of eleven when calculating the anomalous Hall conductivities due to the concomitant change in resistivity. In contrast, these values are only weakly dependent on temperature as shown, e.g., in the $t=1.8 \mathrm{~nm}$ sample in Fig. 3(b).

We have analyzed the data at different temperatures for all studied samples according to the empirical relation $\rho_{x y}$ $=R_{O} \mu_{0} H+R_{S} \mu_{0} M$, and the results of $R_{O}$ and $R_{S}$ at $T=5 \mathrm{~K}$ and $T=300 \mathrm{~K}$ have been plotted as a function of the Fe layer thickness in Fig. 4. In the inset of Fig. 4 we have also represented the thickness dependence of the saturation field compared to VSM results. Regarding the OHE coefficient $R_{O}$, it slightly increases with decreasing thickness and temperature. This increase upon decreasing thickness is in good agreement with the results obtained in much higher conductivity Fe films down to $2 \mathrm{~nm}$ grown by MBE on $\mathrm{MgO}(001)$ (Ref. 25) and on MBE-deposited Fe films down to $6 \mathrm{~nm}$ on GaAs. ${ }^{23}$ Apparently, some dependence $R_{O}\left(\rho_{x x}\right)$ has been reported in polycrystalline $\mathrm{Fe}$ with different impurity contents. ${ }^{26}$ The dependence of $R_{O}$ with temperature has also been reported in Fe polycrystals ${ }^{26}$ and whiskers, ${ }^{27}$ and may be understood within a simple multiband model. Nevertheless, it is important to note that we are calculating $R_{O}$ from the high-field slope of $\rho_{x y}$ versus the applied magnetic field. In a more general situation, this would lead to an effective $R_{O}^{*}$ that is related with $R_{O}$ through the expression $R_{O}^{*}=R_{O}$ $+4 \pi \chi_{\mathrm{hf}}\left[R_{O}(1-N)+R_{S}\right]$, where $N$ is the demagnetization factor and $\chi_{\mathrm{hf}}$ the high-field susceptibility. ${ }^{26}$ In our case, since $N=1$ and $\chi_{\mathrm{hf}} \ll 1$, this leads to $R_{O}^{*} \cong R_{O}$ but this determination has to be taken with caution in the ultrathin limit since there might be changes in both the electronic structure and $N$, and this approximation does not necessarily hold. The focus of this work is the AHE coefficient $R_{S}$ and its dependence on $\rho_{x x}$ but this will be done in terms of the conductivities below. Another important output of the analysis of $\rho_{x y}$ is the saturation field $H_{\text {sat }}$ and from it, the saturation magnetization $M_{S}$ calculated as $M_{S}=\mu_{0} H_{\text {sat }} / 4 \pi$. These values have been repre- 


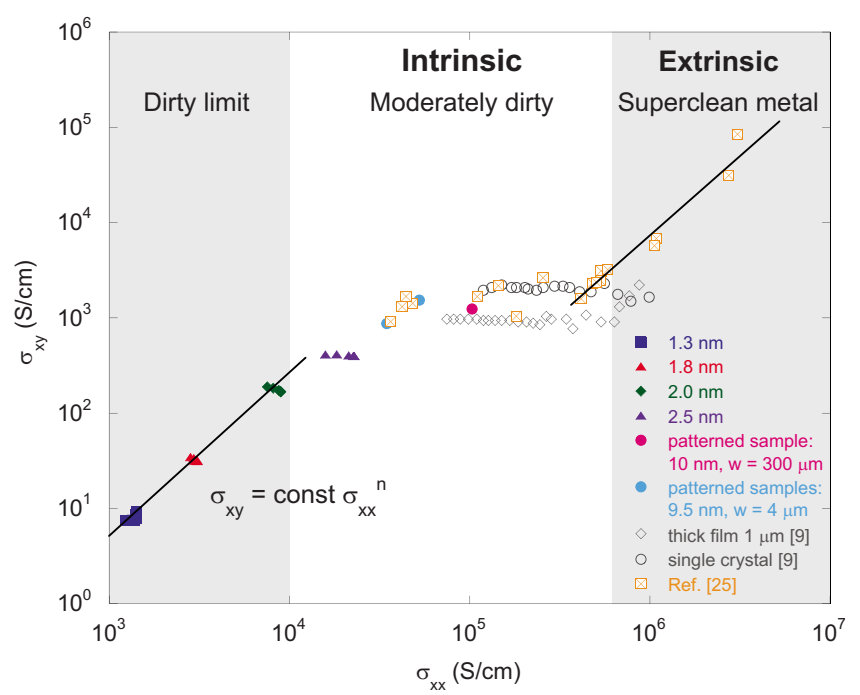

FIG. 5. (Color online) Anomalous Hall conductivity values $\left|\sigma_{x y}\right|$ as a function of longitudinal conductivity $\sigma_{x x}$ in $\mathrm{MgO}(001) / / \mathrm{Fe}(t) / \mathrm{MgO}$ with $t=2.5,2.0,1.8$, and $1.3 \mathrm{~nm}$, and on patterned samples of $t \cong 10 \mathrm{~nm}$. We have included the results for a $1 \mu \mathrm{m}$ thick film and single-crystal specimen of Fe from Ref. 9 and that of MBE-grown Fe thin films down to $2 \mathrm{~nm}$ (Ref. 25). The solid line in the dirty region of conductivities $\left(\sigma_{x x}<10^{4} \mathrm{~S} / \mathrm{cm}\right)$ is the fit according to the scaling relationship $\sigma_{x y}=$ const $\sigma_{x x}^{n}$ with $n$ $=1.66(4)$.

sented in the inset of Fig. 4 and compared to those obtained from VSM magnetometry. As we can observe, the saturation magnetization of our Fe epitaxial structures is within the experimental error in good agreement with that of bulk bcc Fe. There is an apparent slight decrease for the thinnest films but the error bars are very large due to the smaller signal and higher uncertainty in the thickness determination and, therefore, we cannot report this decrease in the magnetic moment as significant. It is important to point out that all our films are ferromagnetic down to $1.3 \mathrm{~nm}$ from room temperature down to $2 \mathrm{~K}$ although in-plane magnetic anisotropy disappears below $1.8 \mathrm{~nm}$. This might be related to an increase in configurational anisotropy over the magnetocrystalline contribution. ${ }^{28}$ We have also observed a decrease in the anisotropic magnetoresistance (AMR) (not reported here) with decreasing thickness, with values of the order of AMR $\cong 0.2 \%$ for $t=1.3 \mathrm{~nm}$, with this being in contrast to that obtained in Ref. 18.

The longitudinal conductivity $\sigma_{x x}$ and the transverse anomalous conductivity $\sigma_{x y}$ were estimated through the relations $\sigma_{x x}=\rho_{x x} /\left(\rho_{x x}^{2}+\rho_{x y}^{2}\right)$ and $\sigma_{x y}=\rho_{x y} /\left(\rho_{x x}^{2}+\rho_{x y}^{2}\right)$, respectively, which can be approximated in our case since $\left|\rho_{x y}\right|$ $\ll \rho_{x x}$ as $\sigma_{x x} \cong 1 / \rho_{x x}$ and $\sigma_{x y} \cong \rho_{x y} / \rho_{x x}^{2}$. We have verified that in all cases this approximation holds. The obtained results from all our samples have been represented in Fig. 5 where we have also included the results from Miyasato et al. ${ }^{9}$ in $\mathrm{Fe}$ single crystals and $1 \mu \mathrm{m}$ thick films, and those from Schad et al. $^{25}$ in Fe films down to $2 \mathrm{~nm}$. Figure 5 has to be compared with Fig. 2 from Ref. 9 and/or Fig. 12 from Ref. 13. The results from Ref. 9 mainly belong in the intrinsic region (moderately dirty regime) with $\sigma_{x x} \sim 10^{4}-5 \times 10^{5} \mathrm{~S} / \mathrm{cm}$ where $\sigma_{x y}=$ const. In this region $\left|\sigma_{x y}\right| \sim 10^{3} \mathrm{~S} / \mathrm{cm}$, which can be mostly assigned to the intrinsic Berry-phase contribution. ${ }^{6,29}$ As seen in Fig. 5, our samples with higher conductivities, i.e., the optically patterned samples of $t$ $\cong 10 \mathrm{~nm}$ and that of $t=2.5 \mathrm{~nm}$ also belong to this regime. At higher conductivities $\sigma_{x x}>5 \times 10^{5} \mathrm{~S} / \mathrm{cm}$, we enter into the extrinsic regime (superclean limit) dominated by the skew scattering mechanism and therefore $\sigma_{x y} \propto \sigma_{x x}$. We have taken the results by Schad et al. ${ }^{25}$ and replotted them in terms of conductivities. These authors plotted $R_{S}$ vs $\rho_{x x}$ (Fig. 5 in Ref. 25) and reported indeed a departure from the $R_{S}$ $\sim \rho_{x x}^{2}$ dependence $\left(\sigma_{x y}=\right.$ const $)$ at high conductivities that lacked theoretical interpretation. Here, we provide a reinterpretation of their results in terms of a crossover between the intrinsic and extrinsic regions of the AHE, according to the unified model put forward by Onoda et al. ${ }^{8}$

Finally, we turn our attention to the dirty regime of relatively low conductivity $\sigma_{x x}<10^{4} \mathrm{~S} / \mathrm{cm}$ where the scaling behavior $\sigma_{x y} \propto \sigma_{x x}^{1.6}$ is apparently universally verified. Our samples with $t=2.0,1.8$ and $1.3 \mathrm{~nm}$ fall into this category. We have fitted our data to the $\sigma_{x y}=$ const $\sigma_{x x}^{n}$ and the fit yields a value of $n=1.66(4)$, in reasonably good agreement to the theory. In Fig. 5 we have included data at all temperatures down to $5 \mathrm{~K}$. At $T<T_{0}$ (see Fig. 1) we have corrected both longitudinal and Hall resistivity values from quantum effects by substracting a $\ln T$ contribution. ${ }^{30}$ Therefore, by reducing the thickness of Fe (001) epitaxial thin films we have managed to engineer both conductivity and AHE, which produce a crossover from the intrinsic moderately dirty region of conductivities to the dirty region of poorly conducting materials. To our knowledge this is the first time that this crossover has been observed and we have also found that a single compound such as bcc Fe can cover all regions of different behavior of the AHE, providing additional support to the unified theory by Onoda et al. ${ }^{8}$ Another important parameter to be considered is the film roughness. We have observed that an increase in roughness leads to a decrease in both $\sigma_{x x}$ and $\sigma_{x y}$. As an example, the $t=2.5 \mathrm{~nm}$ sample reported in Fig. 5 has an rms roughness at the $\mathrm{Fe} / \mathrm{MgO}$ top interface of $0.37 \mathrm{~nm}$ (XRR fit value); this sample having $\sigma_{x y}=413 \mathrm{~S} / \mathrm{cm}$ and $\sigma_{x x}=15794 \mathrm{~S} / \mathrm{cm}$ at room temperature. We have prepared an additional sample with $t=2.5 \mathrm{~nm}$ but $1.12 \mathrm{~nm}$ of $\mathrm{rms}$ roughness. The latter has conductivities of $\sigma_{x y}=125 \mathrm{~S} / \mathrm{cm}$ (70\% decrease) and $\sigma_{x x}=6403 \mathrm{~S} / \mathrm{cm}(60 \%$ decrease). This offers an additional parameter to control both $\sigma_{x y}$ and $\sigma_{x x}$ in Fe thin films either by controlling the growth parameters and/or by patterning the sample and/or the $\mathrm{MgO}$ substrate by, e.g., focused ion beam or electron beam lithographies.

\section{CONCLUSIONS}

In conclusion, we report Hall Effect measurements in ultrathin $\mathrm{MgO}(001) / / \mathrm{Fe}(t) / \mathrm{MgO}$ epitaxial heterostructures with $t=10,2.5,2,1.8$, and $1.3 \mathrm{~nm}$. Our results have been compared with others in similar samples published in the literature and interpreted in terms of a recent unified theory of the anomalous Hall effect in ferromagnets. ${ }^{8}$ We propose 
that the thickness and roughness of the Fe layer are control parameters tuning both the longitudinal and transverse anomalous Hall conductivities, $\sigma_{x x}$ and $\sigma_{x y}$, respectively. We indeed have found upon reducing the Fe thickness a crossover from the intrinsic moderately dirty region of conductivities where $\sigma_{x y}=$ const to the dirty region of poorly conducting materials $\left(\sigma_{x x}<10^{4} \mathrm{~S} / \mathrm{cm}\right)$, where the relation $\sigma_{x y} \propto \sigma_{x x}^{n}$ with $n=1.66(4)$ holds in reasonable agreement with theory. Therefore, a single compound such as bcc Fe can span all regions of different behavior of the AHE, providing additional support to the unified theory by Onoda et al. ${ }^{8}$ We also propose that roughness control either by controlling the growth mode and/or by artificially patterning the sample and/or the $\mathrm{MgO}$ substrate by, e.g., focused ion beam or electron beam lithographies, can be utilized to engineer the magnetotransport properties, especially the AHE.

\section{ACKNOWLEDGMENTS}

Financial support by the Spanish Ministry of Science (MEC) through Projects No. MAT2005-05565-C02 and No. MAT2008-06567-C02 including FEDER funding, and the Aragon Regional Government (Group E26) are acknowledged. The authors thank S. Onoda for a critical reading of the paper and R. Córdoba for lamella preparation with dual beam.
*Corresponding author. Present address: Departamento de Física de la Materia Condensada-INA Facultad de Ciencias, Universidad de Zaragoza, Pedro Cerbuna 12, 50009 Zaragoza, SPAIN. FAX: 34976-761229; morellon@unizar.es; URL: http://fmc.unizar.es/ people/morellon/

${ }^{1}$ R. Karplus and J. M. Luttinger, Phys. Rev. 95, 1154 (1954).

${ }^{2}$ G. Sundaram and Q. Niu, Phys. Rev. B 59, 14915 (1999).

${ }^{3}$ M. V. Berry, Proc. R. Soc. London, Ser. A 392, 45 (1984).

${ }^{4}$ J. Smit, Physica (Amsterdam) 21, 877 (1955); 24, 39 (1958).

${ }^{5}$ J. M. Luttinger, Phys. Rev. 112, 739 (1958).

${ }^{6}$ P. Nozieres and C. Lewiner, J. Phys. (Paris) 34, 901 (1973).

${ }^{7}$ L. Berger, Phys. Rev. B 2, 4559 (1970).

${ }^{8}$ S. Onoda, N. Sugimoto, and N. Nagaosa, Phys. Rev. Lett. 97, 126602 (2006).

${ }^{9}$ T. Miyasato, N. Abe, T. Fujii, A. Asamitsu, S. Onoda, Y. Onose, N. Nagaosa, and Y. Tokura, Phys. Rev. Lett. 99, 086602 (2007).

${ }^{10}$ T. Fukumura, H. Toyosaki, K. Ueno, M. Nakano, T. Yamasaki, and M. Kawasaki, Jpn. J. Appl. Phys., Part 2 46, L642 (2007), and references therein.

${ }^{11}$ S. Onoda, N. Sugimoto, and N. Nagaosa, Phys. Rev. B 77, 165103 (2008).

${ }^{12}$ A. Fernández-Pacheco, J. M. De Teresa, J. Orna, L. Morellon, P. A. Algarabel, J. A. Pardo, and M. R. Ibarra, Phys. Rev. B 77, 100403(R) (2008).

${ }^{13}$ D. Venkateshvaran, W. Kaiser, A. Boger, M. Althammer, M. S. Ramachandra Rao, S. T. B. Goennenwein, M. Opel, and R. Gross, Phys. Rev. B 78, 092405 (2008).

${ }^{14}$ C. Li and A. J. Freeman, Phys. Rev. B 43, 780 (1991).

${ }^{15}$ S. Yuasa, T. Nagahama, A. Fukushima, Y. Suzuki, and K. Ando, Nature Mater. 3, 868 (2004).

${ }^{16}$ For review, see P. A. Lee and T. V. Ramakrishnan, Rev. Mod. Phys. 57, 287 (1985); G. G. Aliev, V. K. Dugaev, and J. Barnás, in Encyclopedia of Nanoscience and Nanotechnology, edited by H. S. Nalwa (American Scientist, Stevenson Ranch, CA, 2004), Vol. 4, p. 587.

${ }^{17}$ C. Liu, Y. Park, and S. D. Bader, J. Magn. Magn. Mater. 111, L225 (1992).

${ }^{18}$ C. Martinez-Boubeta, Ll. Balcells, and A. Cebollada, Appl. Phys. Lett. 88, 132511 (2006).

${ }^{19}$ R. Moons, S. Blässer, J. Dekoster, A. Vantomme, J. De Wachter, and G. Langouche, Thin Solid Films 324, 129 (1998).

${ }^{20}$ P. Granberg, P. Isberg, T. Baier, B. Hjörvarsson, and P. Nordblad, J. Magn. Magn. Mater. 195, 1 (1999).

${ }^{21}$ J. Orna, L. Morellon, P. A. Algarabel, J. A. Pardo, S. Sangiao, C. Magen, E. Snoeck, J. M. De Teresa, and M. R. Ibarra, IEEE Trans. Magn. 44, 2862 (2008).

${ }^{22}$ E. H. Sondheimer, Adv. Phys. 1, 1 (1952).

${ }^{23}$ M. Rubinstein, F. J. Rachford, W. W. Fuller, and G. A. Prinz, Phys. Rev. B 37, 8689 (1988).

${ }^{24}$ G. Wedler, C. M. Schneider, A. Trampert, and R. Koch, Phys. Rev. Lett. 93, 236101 (2004).

${ }^{25}$ R. Schad, P. Beliën, G. Verbanck, V. V. Moshchalkov, and Y. Bruynseraede, J. Phys.: Condens. Matter 10, 6643 (1998).

${ }^{26}$ C. M. Hurd, The Hall Effect in Metals and Alloys (Plenum, New York, 1972), and references therein.

${ }^{27}$ P. N. Dheer, Phys. Rev. 156, 637 (1967).

${ }^{28}$ C. Martínez Boubeta, C. Clavero, J. M. Garcia-Martin, G. Armelles, A. Cebollada, L. Balcells, J. L. Menendez, F. Peiro, A. Cornet, and M. F. Toney, Phys. Rev. B 71, 014407 (2005).

${ }^{29}$ Y. Yao, L. Kleinman, A. H. MacDonald, J. Sinova, T. Jungwirth, D.-S. Wang, E. Wang, and Q. Niu, Phys. Rev. Lett. 92, 037204 (2004).

${ }^{30}$ P. Mitra, R. Mitra, A. F. Hebard, K. A. Muttalib, and P. Wolfle, Phys. Rev. Lett. 99, 046804 (2007). 